Revue d'histoire de l'Amérique française

REVUE D.HISTOIRE DE L'AMÉRIQUE FRANÇAISE

\title{
Industries, nuisances et définition de l'espace urbain. L’industrie papetière à Trois-Rivières (1910-1925)
}

\section{Carole Payen}

Volume 68, numéro 3-4, hiver-printemps 2015

URI : https://id.erudit.org/iderudit/1033636ar

DOI : https://doi.org/10.7202/1033636ar

Aller au sommaire du numéro

Éditeur(s)

Institut d'histoire de l’Amérique française

ISSN

0035-2357 (imprimé)

1492-1383 (numérique)

Découvrir la revue

Citer cet article

Payen, C. (2015). Industries, nuisances et définition de l'espace urbain. L'industrie papetière à Trois-Rivières (1910-1925). Revue d'histoire de l'Amérique française, 68(3-4), 241-270. https://doi.org/10.7202/1033636ar
Résumé de l'article

Trois-Rivières connut, grâce aux industries papetières, une croissance fulgurante au début du $\mathrm{XX}^{\mathrm{e}}$ siècle. Cependant, ces industries furent génératrices de nombreuses nuisances et firent naître la controverse. Celle-ci, qui s'exprime dans de multiples sources, administratives, judiciaires, médiatiques, industrielles, offre la possibilité d'étudier les discours liés à la ville, et notamment les questions d'arbitrage et de définition de l'intérêt général (croissance économique vs salubrité), mais également de percevoir des discours sur le territoire, l'espace urbain, ses limites, et le(s) « territoire(s) acceptable(s) » de la pollution industrielle au début du XX $\mathrm{X}^{\mathrm{e}}$ siècle. 


\title{
Industries, nuisances et définition de l'espace urbain. L'industrie papetière à Trois-Rivières (I9IO-I925)
}

\author{
Carole Payen \\ Pôle de l'histoire environnementale \\ Université de Namur (PolleN)
}

RÉSUMÉ • Trois-Rivières connut, grâce aux industries papetières, une croissance fulgurante au début du $X X^{e}$ siècle. Cependant, ces industries furent génératrices de nombreuses nuisances et firent naître la controverse. Celle-ci, qui s'exprime dans de multiples sources, administratives, judiciaires, médiatiques, industrielles, offre la possibilité d'étudier les discours liés à la ville, et notamment les questions d'arbitrage et de définition de l'intérêt général (croissance économique vs salubrité), mais également de percevoir des discours sur le territoire, l'espace urbain, ses limites, et le(s) «territoire(s) acceptable(s)» de la pollution industrielle au début du $X X^{e}$ siècle.

ABSTRACT - At the onset of the 20th century, Three-Rivers witnessed the rapid growth of its urban center due to the establishment of a paper industry. However, this industry caused nuisance and pollution, which in turn triggered a controversy that was documented in multiple sources, i.e. legal, administrative and industrial archives as well as newspapers. These sources provide an opportunity to study discourses about the city, in particular questions of arbitration and the definition of common good (economic growth vs. public health), and discourses on territory, urban space, boundaries, and "territories deemed acceptable" for industrial pollution in the early 20th century.

\section{INTRODUCTION}

La fin du XIX et le début du XX ${ }^{\mathrm{e}}$ siècle voient, au Québec, l'émergence de plusieurs centres urbains, notamment grâce à l'implantation de grandes 
industries. Certaines villes naissent avec la découverte de ressources et l'établissement d'industries qui s'installent sur un territoire et instiguent de véritables politiques de développement urbain ${ }^{1}$. À Shawinigan, par exemple, ville nouvelle créée sous l'impulsion de la Shawinigan Water and Power Co., les principales industries de la ville jouent un rôle important dans les affaires publiques, notamment en termes de planification de l'espace urbain, au début du $\mathrm{XX}^{\mathrm{e}}$ siècle ${ }^{2}$. D'autres villes, comme TroisRivières, étaient, jusqu'à la fin du XIX siècle, de petits centres régionaux et connurent une croissance fulgurante grâce à ces implantations industrielles. La relation directe entre la présence de communautés humaines et l'établissement d'industries est une question ancienne, puisque déterminante dans le développement des centres de peuplement au Québec ${ }^{3}$. Cependant, peu d'études approchent le vécu des communautés anciennes et le potentiel conflictuel de la confrontation de ces citoyens au développement industriel de leur ville. Or ce sont des changements radicaux auxquels ont dû faire face ces villes à l'arrivée, en leur sein, de nouvelles industries. Celles-ci en modifient profondément le visage et l'identité.

Fondée en 1634, Trois-Rivières se développe à un rythme lent jusqu'au milieu du XIX ${ }^{\mathrm{e}}$ siècle. L'industrie de la coupe du bois lui fait alors vivre un premier essor, qui sera cependant sans commune mesure avec le bouleversement engendré par l'installation de l'industrie papetière, début $\mathrm{XX}^{\mathrm{e}}$ siècle, et vaudra à la ville son surnom de "capitale mondiale du papier». L'historiographie trifluvienne fait, bien sûr, la part belle au fleuron de l'industrie de la ville durant près d'un demi-siècle, aucun ouvrage sur la cité ne pouvant se passer d'un large chapitre dédié à l'industrie papetière. Certes, certains ouvrages citent les problèmes liés aux émanations des usines de papiers, mais aucun n’approche ce phénomène au-delà de quelques citations de presse pour s'intéresser à la gestion qui a entouré ces nuisances, et aux éventuels impacts négatifs de l'influente industrie sur la ville dans laquelle elle s'installe.

1. Robert Fortier, dir., Villes industrielles planifiées (Montréal, Boréal, 1996).

2. François Guérard, La santé publique dans deux villes du Québec de 1887 à 1939. Trois-Rivières et Shawinigan, Thèse de doctorat (histoire), Université du Québec à Montréal, 1993, 45-49; Pierre Lanthier et Normand Brouillette, «Shawinigan Falls de 1898 à 1930. L'émergence d'une ville industrielle au sein du monde rural», Revue d'histoire urbaine, 19, 1 (juin 1990): 42-55; Normand Brouillette, «Le rôle de la Shawinigan Water and Power Co. dans la structuration de l'espace urbain shawiniganais, 1898-1921", Cahiers de géographie du Québec, 34, 92 (1990): 197-208.

3. Jean-Charles Falardeau, «L'évolution de nos structures sociales», dans Marcel Rioux et Yves Martin, dir., La société canadienne française (Montréal, Les Éditions Hurtubise, 1971), 119-133. 
De manière générale, le Québec manque d'études ${ }^{4}$ telles que celles effectuées sur l'Europe ou sur les États-Unis sur la gestion des pollutions industrielles et les conflits en découlant ${ }^{5}$. Le cas trifluvien permet une analyse détaillée. Comment les industries papetières ont-elles modifié le territoire urbain, son identité, ses délimitations? Trouve-t-on trace de voix dissonantes et réfractaires à ce développement industriel soudain de la cité ? Comment, au début du $\mathrm{XX}^{\mathrm{e}}$ siècle, les nuisances importantes liées aux industries papetières sont-elles abordées et gérées au sein de la cité trifluvienne?

Le cadre géographique de cette étude comprend la municipalité actuelle de Trois-Rivières, c'est-à-dire les territoires de Trois-Rivières et du Capde-la-Madeleine. Ne formant aujourd'hui qu'une seule et même municipalité, les deux agglomérations, administrativement distinctes jusqu'en 2002, ont un destin similairement lié à l'industrie papetière, tout en présentant des caractéristiques différentes influant sur la manière dont chacune a géré l'impact industriel. L'année 1910 correspond à l'arrivée de la première usine de pâtes et papiers sur ce territoire. Celle de 1925 marque en quelque sorte la fin d'une période de transition: les principaux changements au niveau démographique et urbain en lien direct avec les nouvelles industries ont eu lieu, les idées réfractaires se sont exprimées et le problème des émanations provenant des usines est en voie d'être résolu, partiellement du moins.

Les sources mises ici à contribution sont de diverses origines ${ }^{6}$. Tant les archives législatives qu’administratives ont été consultées: correspondances et rapports du Conseil provincial d'hygiène, Statuts de la province de

4. Claire Poitras, «L'histoire urbaine environnementale au Québec. Un domaine de recherche en émergence», Globe: revue internationale d'études québécoises, 9, 1 (2006): 93-111.

5. Pour citer quelques exemples: Christoph Bernhardt et Geneviève Massard-Guilbaud, dir., Le démon moderne. La pollution dans les sociétés urbaines et industrielles d'Europe (Clermont-Ferrand, Presses Universitaires Blaise-Pascal, 2002), 465 p.; Geneviève Massard-Guilbaud, Histoire de la pollution industrielle. France, 1789-1914 (Paris, Éditions de l'EHESS, 2010), 403 p.; Thomas Leroux et Michèle Letté, dir., Débordements industriels. Environnement, territoire et conflit. XVIII'-XXI ${ }^{e}$ siècle (Rennes, Presses Universitaires de Rennes, 2013), 402 p. ; Isabelle Parmentier, Histoire de l'environnement en Pays de Charleroi, 1730-1830. Pollution et nuisances dans un paysage en voie d'industrialisation (Bruxelles, Académie royale de Belgique, 2008), 410 p.; Peter Thorsheim, Inventing Pollution. Coal, Smoke and Culture in Britain since 1800 (Athens, Ohio University Press, 2006), 307 p. ; Peter Brimblecombe et Christian Pfister, dir., The Silent Countdown. Essays in European Environmental History (Berlin et Heidelberg, Springer-Verlag, 1990), 265 p.; Martin V. Melosi, Effluent America. Cities, Industry, energy, and the Environment (Pittsburgh, University of Pittsburgh Press, 2001), 325 p. ; Chad Montrie, To Save the Land and People. A History of Opposition to Surface coal Mining in Appalachia (Chapel Hill-London, The University of North Carolina Press, 2003), 245 p.

6. Recherches effectuées à l'occasion de deux séjours au Québec, dont un séjour de trois mois financé sur bourse de recherche pour chercheurs extérieurs du Québec par BAnQ (Bibliothèque et Archives nationales du Québec) de mai à juillet 2012. 
Québec, chartes et règlements municipaux, procès-verbaux des réunions des Conseils municipaux du Cap-de-la-Madeleine et de Trois-Rivières (de 1910 à 1925). Le Fonds de la Consolidated Bathurst Incorporated, qui a racheté la Wayagamack Pulp and Paper Co. et la St. Maurice Paper Co., a également été exploité, ainsi que les archives des cours supérieures de TroisRivières et de Montréal. Enfin, trois journaux trifluviens, Le Bien Public, Le Courrier et Le Nouvelliste, ont été parcourus afin d'identifier les articles relatifs aux pulperies. À cela s'ajoutent quelques sources complémentaires et secondaires ainsi qu'une riche bibliographie locale.

En quinze ans, ce sont quatre entreprises de production de pulpe et de papier qui s'implantent sur le territoire de Trois-Rivières et du Cap-de-laMadeleine. La population bondit de 11445 habitants en 1901 à 44198 en $1931^{7}$. La première partie de notre étude s'intéressera à l’impact territorial et démographique de ce développement soudain. La deuxième partie sera ensuite dédiée à l'étude des discours entourant ce développement industriel. En effet, malgré l'indépendance toute relative des autorités municipales par rapport aux industriels ${ }^{8}$, l'installation de ces entreprises à Trois-Rivières n'est pas sans provoquer des débats, notamment à la suite des émanations provenant des usines de pâtes et papiers. Cette controverse, s'exprimant dans la presse, lors des réunions des autorités municipales, mais également devant les tribunaux, laisse percevoir une réflexion sur la place de l'industrie en ville, et plus largement sur la ville en tant que telle, sur les fonctions qu'elle doit remplir, les objectifs qu'elle se donne, sur ce qui rassemble les habitants et les droits qui accompagnent ce statut d'habitants, de citoyens. Ces débats sont aussi l'occasion d'approcher les discussions sur la dangerosité des rejets industriels, dont traitera la troisième partie de cette étude. Certaines industries, dont celle des pâtes et papiers, constituent de véritables nuisances publiques au début du $\mathrm{XX}^{\mathrm{e}}$ siècle, suscitant des interrogations quant à l'impact industriel sur la qualité de l'air, la salubrité des eaux et sur les organismes vivants qui en dépendent.

7. Populations de Trois-Rivières et de Cap-de-la-Madeleine additionnées. René Hardy et Normand Séguin, Forêts et société en Mauricie. La formation de la région de Trois-Rivières 1830-1930 (Montréal, Boréal Express/Musée national de l'Homme, 1984), 196.

8. Pour un exposé chronologique de l'action des différents acteurs, et notamment de la gestion par les municipalités, voir Carole Payen, " "Ça sent le jambon...". Trois-Rivières et l'essor de l'industrie papetière (1910-1925)", Isabelle Parmentier et Olivier Servais, dir., Le pouvoir des riverains : résistances, accommodations, illusions? Histoire et anthropologie des mobilisations citoyennes (XVIII ${ }^{e}$-XXI $I^{e}$ siècles), à paraître, 2015. 


\section{EXPLOSION DÉMOGRAPHIQUE ET DÉVELOPPEMENT URBAIN}

L'industrialisation a joué, au Québec et en Amérique du Nord en général, un rôle primordial dans la définition des territoires. Les industries participent à redessiner l'organisation territoriale dans une logique d'exploitation des ressources et d'appropriation de nouvelles contrées, par exemple par l'aménagement de voies de communication. C'est notamment dans ses retombées sur les populations autochtones que la thématique est abordée par les historiens et anthropologues, évaluant l'influence, à court et moyen terme, de l'implantation d'infrastructures (barrages hydroélectriques, lignes à haute tension, réseaux routiers...) sur la vie des populations autochtones présentes antérieurement sur le territoire ou dans son voisinage ${ }^{9}$. Est parfois traitée la question de la résistance à l'industrialisation et à l'urbanisation dans le cadre de la construction de grands ouvrages hydroélectriques ${ }^{10}$. Ces derniers, très visibles dans le paysage, ont des impacts profonds et durables, devenant des figures emblématiques du développement québécois. Certaines études s’intéressent au développement de ces villes, sinon mono-industrielles, du moins créées pour et par l'industrie, qui subissent un brutal coup d'arrêt avec la fermeture de "l'entreprise mère», devant alors se diversifier ou chercher de nouvelles vocations, au risque de tout simplement disparaitre ${ }^{11}$. Trois-

9. À titre d'exemples: Robert Lanari, dir., «Les Inuits, les Premières Nations et le développement minier", Recherches amérindiennes au Québec, 40, 3 (numéro thématique, 2010); Stéphane Savard, «Les communautés autochtones du Québec et le développement hydroélectrique: un rapport de force avec l'État, de 1944 à aujourd'hui ", Recherches amérindiennes au Québec, 39, 1-2 (2009): 47-60; Daniel Rueck, "When bridges become barriers. Montreal and Kahnawake Mohawk territory», dans Stéphane Castonguay et Michèle Dagenais, Metropolitan Natures. Environmental Histories of Montreal (Pittsburgh, University of Pittsburgh Press, 2011), 228-244; Mélanie Chaplier, «Le conflit à la baie James. Pour une anthropologie de la nature dans un contexte dynamique", Civilisations [En ligne], 55 (2006): 103-115; David Gilles, «Pollution environnementale, pollution sociale, enjeux d'une nouvelle réflexivité? Le droit minier face aux communautés locales et "autochtones" au Québec et au Canada», dans Hervé Pujol, dir., Tristes mines. Impacts environnementaux et sanitaires de l'industrie extractive (Bordeaux, Les Études Hospitalières Édition, 2014), 285-303.

10. Victor Tremblay, La tragédie du Lac Saint-Jean (Chicoutimi, Éditions Science moderne, 1979), 231 p. ; David Massell, Amassing Power: J.B. Duke and the Saguenay River, 1897-1927 (Montréal et Durham, McGill-Queen’s University Press et the Forest History Society, 2000), 301 p.; Louis-Raphaël Pelletier, "The destruction of the Rural Hinterland. Industrialization of Landscapes in Beauharnois County", dans S. Castonguay et M. Dagenais, Metropolitan Natures..., op. cit., 245-263.

11. Claude Bellavance et François Guérard, «Ségrégation résidentielle et morphologie urbaine, le cas de Shawinigan, 1925-1947", Revue d'histoire d'Amérique française, 46, 4 (printemps 1993): 577-605; Thierry Haroun, Murdochville. Histoire d'une fermeture (Trois-Pistoles, Éditions Trois-Pistoles, 2005), 177 p. ; Olivier Servais, «Faut-il fermer Murdochville?», La revue nouvelle, 1-2 (janvier-février 2004): 52-59; Rex A. Lucas et Lorne Tepperman, Minetown, Milltown, Railtown. Life in Canadian communities of single industry (Don. Mills, Ont., Anniversary Edition, Oxford University Press, 2008), 448 p. ; Robert M. Bone, "Resource Towns in The Mackenzie Basin», Cahiers de géographie du Québec, 42, 116 (1998): 249-259; Laurent Deshaies, Analyse spatiale de la croissance et de la décroissance des villes minières canadiennes, Thèse 
Rivières subit elle aussi de plein fouet l'arrivée de l'industrie papetière qui engendre de multiples changements dans le développement urbain des cités trifluvienne et madelinoise: détournement de rues, augmentation de la population, développement des quartiers ouvriers et modification de la dynamique générale des quartiers.

La position géographique de Trois-Rivières et les avantages qui en découlent attirent les industries papetières à l'aube du $\mathrm{XX}^{\mathrm{e}}$ siècle ${ }^{12}$. La ville offre en effet un accès tant aux ressources de l'arrière-pays, via la rivière Saint-Maurice, qu'aux marchés internationaux, via le Saint-Laurent et le chemin de fer. La proximité de la Shawinigan Water and Power Company (1898) assure une énergie abondante à bas prix. Ajoutons à cela l'embargo qui, à partir de 1910, interdit aux entreprises d'exporter du bois non transformé ${ }^{13}$, et voici les villes québécoises se lançant dans une compétition. Laquelle offrira le plus d'avantages pour attirer les nouvelles industries, essentiellement américaines, qui cherchent à s'implanter dans les régions regorgeant de matières ligneuses? Trois-Rivières et Cap-de-la-Madeleine ne font pas exception et mènent de véritables politiques urbaines en ce sens ${ }^{14}$.

Les entreprises, quant à elles, savent jouer de la concurrence entre les municipalités. Un bon exemple reste l'extension du moulin à scie et l'installation de la manufacture de pulpe de la Grès Falls \& Union Bag \& Paper, future St. Maurice Paper Co. Le moulin était situé dans la municipalité de Trois-Rivières, sur les rives de la rivière Saint-Maurice, au nord-est de la ville. En août 1910, l'industriel veut agrandir ses installations et réclame plusieurs faveurs à la ville ${ }^{15}$ : des avantages fiscaux, le détournement de la rue des Pins, l'alimentation gratuite en eau et un bonus de 25000 \$. Mais le Conseil, en raison de la situation financière délicate de la ville après le

de doctorat (géographie), Université Laval, 1977; John H. Bradbury, «The Rise and Fall of the «Fourth Empire of the St. Lawrence» : the Québec-Labrador Iron Ore Mining Region », Cahiers de géographie du Québec, 29, 78 (1985): 351-364.

12. Avantages quelque peu relativisés par l'étude de Ronald Rudin, The Development of Four Quebec Towns, 1840-1914, a Study of Urban and Economic Growth in Quebec, Thèse de doctorat (histoire), York University, 1977, 66-85.

13. Alain Dion, L'industrie des pâtes et papiers en Mauricie (1887-1929), mémoire de maîtrise (études québécoises), Université du Québec à Trois-Rivières, 1981, 7-14, 36-37; Jean-Pierre Charland, Les pâtes et papiers au Québec 1880-1980. Technologies, travail et travailleurs (Québec, Institut québécois de recherche sur la culture, 1990), 62-69.

14. R. Hardy et N. Séguin, Forêt et société en Mauricie..., op. cit., 197-198; R. Hardy et N. Séguin, dir., Histoire de la Mauricie (Sainte-Foy, Les Presses de l'Université Laval, 2004), 573-583; J.-P. Charland, Les pâtes et papiers au Québec..., op. cit., 70-71.

15. "The Gres Falls \& Union Bag \& Paper Co. vs Le Conseil de ville des Trois-Rivières », Le Courrier, 14 février 1913. 
Grand Incendie de 1908, ne lui accorde pas le bonus. Quelque temps plus tard, les journaux annoncent l'établissement de la Grès Falls au Cap-dela-Madeleine, où elle participera grandement au développement de la municipalité ${ }^{16}$.

Ces cas de négociations tendues sont courants et, dans le cas de TroisRivières, connaissent un nouvel épisode lors de l'installation et des négociations d'exemption fiscale de la St. Lawrence Paper Mills (d'abord nommée «Three Rivers Pulp \& Paper Co.»)17. La population se montre de plus en plus hostile aux largesses accordées par la municipalité, lesquelles mettent en péril la santé financière de la ville. Une requête, relayant «la protestation de quelques milliers de citoyens contre le projet», est déposée auprès du conseil municipa ${ }^{18}$. Les protestataires ne sont pas seulement inquiets à propos des dépenses annoncées pour l'aménagement de l'aqueduc, le drainage, le pavage et de la hausse des taxes qui pèseront conséquemment sur les contribuables. Ils s'inquiètent aussi du détournement de la rue Notre-Dame: cette construction menace de bloquer l'expansion du port. Cependant, la St. Lawrence exerce encore une fois des pressions: d'autres villes lui ont offert des terrains gratuits et «autres avantages ${ }^{19}$. L'usine est construite en 1922, avec le détournement annoncé de la rue Notre-Dame ${ }^{20}$, mais le contentieux avec la municipalité à propos des taxes ne trouvera une issue qu'en 1926 grâce à l'intervention du gouvernement québécois en faveur de l'entreprise ${ }^{21}$. Ces avantages demandés par les industries, en plus de mettre en évidence les pressions exercées sur les villes pour en tirer un maximum d'avantages, révèlent une des principales difficultés liées à l'installation d'usines à Trois-Rivières : ces dernières doivent s'insérer dans un tissu urbain préexistant ce qui demande parfois un réaménagement de leurs abords.

La première industrie à s'implanter dans l'actuelle municipalité trifluvienne est la Grès Falls, future St. Maurice Paper Co., qui, après les tergiversations déjà décrites, s’installe en 1910 à l'est du Cap-de-la-Madeleine, à côté du Sanctuaire de Notre-Dame du Cap. En 1913, la Wayagamack Pulp and Paper Co. commence ses activités dans l'île de la Potherie, sur

16. A. Dion, L'industrie des pâtes et papiers..., op. cit., 146-147.

17. "La "Three Rivers Pulp and Paper" commencera à construire bientôt », Le Nouvelliste, 12 février 1921, 2 ; "Conditions pour la construction de la "Three Rivers Pulp and Paper" ", Le Nouvelliste, 4 mai 1922, 3 ; A. Dion, L'industrie des pâtes et papiers..., op. cit., 148-154.

18. "L’Établissement de ce moulin n'aura pas été sans difficulté», Le Nouvelliste, 5 mai 1922, 3.

19. "Conditions pour la construction de la "Three Rivers Pulp \& Paper" ", Le Nouvelliste, 4 mai 1922, 3.

20. "Les travaux commenceront le $1^{\text {er }}$ de juin », Le Nouvelliste, 22 mai 1922, 1.

21. R. Hardy et N. Séguin, Forêts et société en Mauricie..., op. cit., 197-198. 
la rivière Saint-Maurice. Les années 1920 verront, d'une part, l'installation de la Canadian International Paper (CIP) sur le vaste terrain situé à l'est du territoire trifluvien, à l'embouchure du Saint-Maurice, et d'autre part l'implantation de la St. Lawrence Paper à l'ouest de la municipalité (figure 1). C'est donc un véritable encerclement de la ville par l'industrie papetière qui se dessine en ce début de $\mathrm{XX}^{\mathrm{e}}$ siècle. Et cet encerclement ne fait pas que des heureux: "A une usine puante établie ici depuis plus de dix ans, est venue $[s i c]$ se joindre une deuxième, puis une troisième. Nous sommes aujourd'hui menacés d'être complètement encerclés par une ceinture de gaz malodorants ${ }^{22}$.» Mais cette ceinture industrielle va bien vite être rejointe, voire dépassée, par le bâti, puisque ces implantations vont entraîner des mouvements de population, tant de l'extérieur vers l'intérieur de l'agglomération, qu'à l'intérieur même des municipalités, modifiant parfois radicalement les abords immédiats des usines et les quartiers adjacents.

\section{Trois-Rivières}

À la fin du XIX ${ }^{\mathrm{e}}$ siècle, Trois-Rivières est essentiellement constituée des quartiers Sainte-Ursule et Saint-Louis, et des quartiers moins bâtis, NotreDame, au nord, et Saint-Philippe, à l'ouest ${ }^{23}$. Dans les premières décennies $\mathrm{du} \mathrm{XX}^{\mathrm{e}}$ siècle, les travailleurs, qui affluent notamment pour travailler dans les pulperies, peuplent le quartier Notre-Dame, y faisant surgir un vaste secteur ouvrier ${ }^{24}$. La demande en logements explose, le quartier s'étend:

Notre-Dame, Trois-Rivières - Les déménagements sont commencés et sont très nombreux. Il $\mathrm{y}$ a tout près d'un tiers de la population qui déménage. Cela est dû à la hausse des loyers qui est générale. C'est surtout dans la partie du nord de la ville dans le quartier Notre-Dame que les déplacements sont les plus nombreux. C'est un quartier manufacturier où les logements sont en partie très étroits et insalubres ${ }^{25}$.

À l'est et au nord-est de la ville, les résidences ouvrières rejoignent les usines $^{26}$. Ainsi, le nombre et la valeur des permis de construction à Trois-

22. "Notre Société médicale et ces gaz infects», Le Bien Public, 23 novembre 1922, 1.

23. Samuel Rousseau, Trois-Rivières: une ville dépossédée de sa région. Étude de morphologie urbaine, mémoire de maîtrise (aménagement), Université de Montréal, 2008, 106.

24. «Des centaines de paroissiens de Notre-Dame sont des ouvriers de la Wayagamack Pulp and Paper», Le Nouvelliste, 30 octobre 1926, 2.

25. Le Bien Public, 20 mai 1914, 8.

26. Alain Gamelin, René Hardy et al., Trois-Rivières illustrée (Trois-Rivières, Corporation des fêtes du $350^{\mathrm{e}}$ anniversaire, 1984), 79-82. 
Figure I

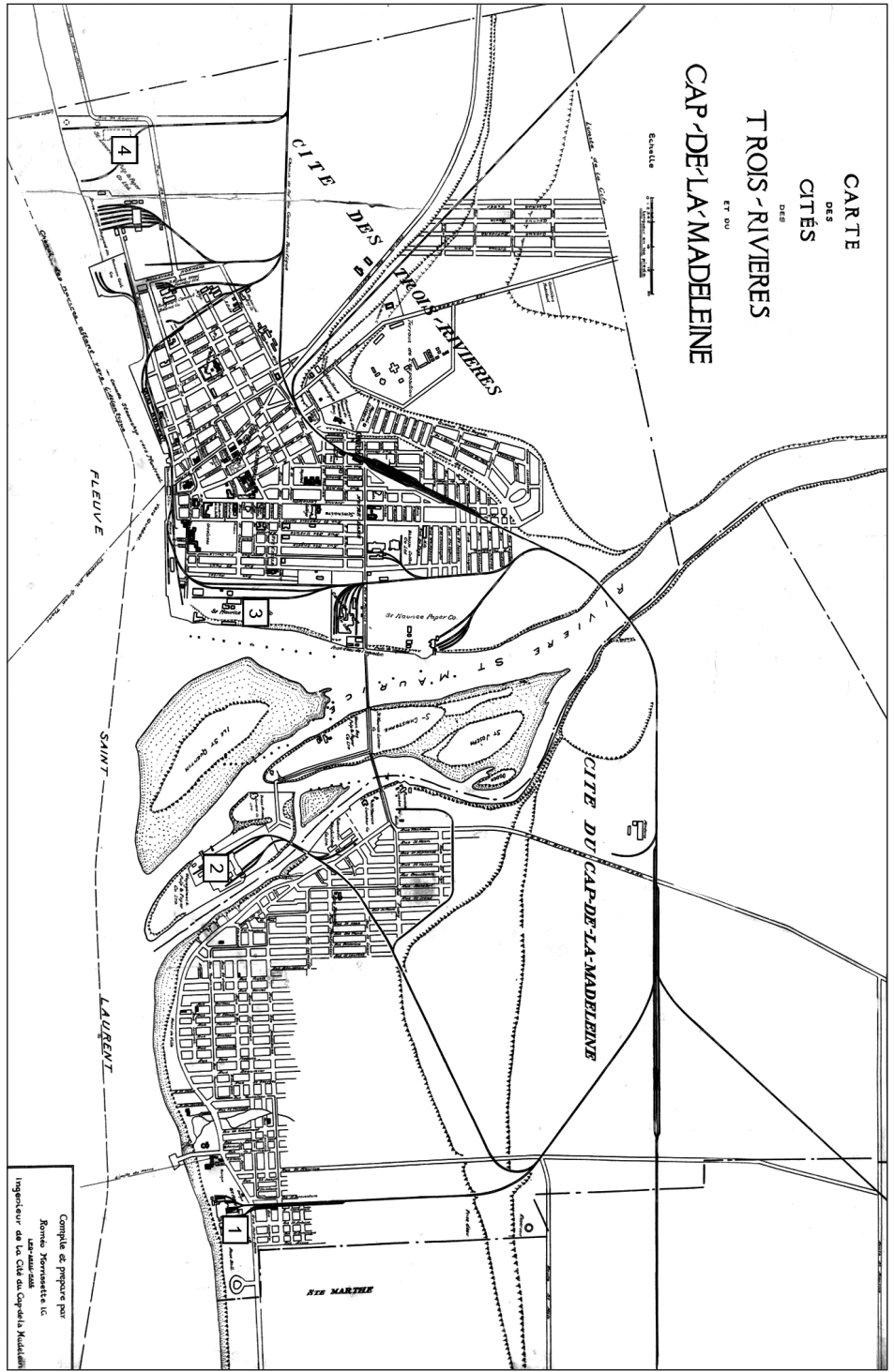

Plan présentant les implantations des industries de pâtes et papiers à Trois-Rivières et au Cap-dela-Madeleine en 1930

I. St. Maurice Paper Co. - 2. Wayagamack Pulp and Paper - 3. Canadian International Paper - 4. St. Lawrence Paper Mills.

Source: Bibiliothèque et Archives Canada (BAC), Carte des cités des Trois-Rivières et du Cap-dela-Madeleine. Compilée et préparée par Roméo Morrissette, I.C., Ingénieur de la cité du Cap- dela-Madeleine, (1930). 
Rivières ne cessent d'augmenter entre 1910 et $1928^{27}$. La ville grandit, s'étend vers l'ouest (création de la paroisse Saint-Philippe, 1909), vers l'est (Notre-Dame-des-Sept-Allégresses, 1911; Sainte-Cécile, 1912) puis vers le nord dans les années 1920 (Très-Saint-Sacrement, 1926; Saint-Françoisd'Assise, 1927) ${ }^{28}$. En 1931, les quartiers Saint-Philippe et Notre-Dame sont décrits comme assez semblables: quartiers populaires, dont les trois quarts de la population environ travaillent dans le secteur secondaire, la transformation des matières premières. Sainte-Ursule et Saint-Louis restent équilibrés entre secteurs secondaires et tertiaires ${ }^{29}$. À l'aube du $\mathrm{XX}^{\mathrm{e}}$ siècle, Trois-Rivières comptait 9981 habitants. En 1931, elle en compte $35450^{30}$. Parmi cette population, en 1925, 2750 ouvriers travaillent dans les 3 usines de production de pâtes et papiers trifluviennes ${ }^{31}$. Le Nouvelliste affirme que «en 1936 l'industrie du papier a fait directement ou indirectement vivre la moitié de la population de Trois-Rivières ${ }^{32}$ ».

Les plans de Trois-Rivières en 1910 et 1930 permettent d'appréhender l'ensemble des impacts de l'arrivée de la grande industrie (figures 2 et 3 ). À l'ouest, la St. Lawrence Paper a provoqué le détournement de la rue Notre-Dame. Le quartier Saint-Philippe s'est développé pour peu à peu assurer la jonction entre l'usine et le centre-ville. La CIP, à l'est, sur les bords du Saint-Maurice, a entraîné le développement des quartiers NotreDame et Sainte-Cécile, tissant un réseau urbain continu entre cette partie de la ville, les quartiers anciens des Ursulines et Saint-Louis et la Wabasso Cotton Co. au nord. Les rails du Canadien Pacifique ont été plusieurs fois prolongés pour assurer la liaison directe avec les plus grandes industries de la ville: les trois papeteries (bien que les rails de la Wayagamack arrivent dans l'île de la Potherie via le Cap-de-la-Madeleine), l'usine textile de la Wabasso Cotton Co., la Three Rivers Shipyard Ltd. et l'ensemble des installations portuaires.

Mais l'augmentation de la population ne se marque pas que dans l'étendue du tissu urbain: l'habitat s'est également considérablement den-

27. René Verrette, Les idéologies de développement régional. Le cas de la Mauricie 1850-1950 (Sainte-Foy, Les Presses de l'Université Laval, 1999), 186.

28. A. Gamelin, R. Hardy et al., Trois-Rivières illustrée, op. cit., 181.

29. Guy et Richard Cossette Trépanier, Trois-Rivières et ses quartiers: 1851-1931: données relatives à la population, à la structure professionnelle et à l'occupation de l'espace (Trois-Rivières, Société de conservation et d'animation du patrimoine de Trois-Rivières, 1984), 47.

30. R. Hardy et N. Séguin, Forêts et Société en Mauricie..., op. cit., 196.

31. A. Dion, L'industrie des pâtes et papiers..., op. cit., 28.

32. "L'industrie du papier et de la pulpe. Trois-Rivières, le plus grand centre de production", Le Nouvelliste, 27 février 1937, 12. 
Figure 2

Plan de la ville de Trois-Rivières en 1910: Quartiers et principales industries

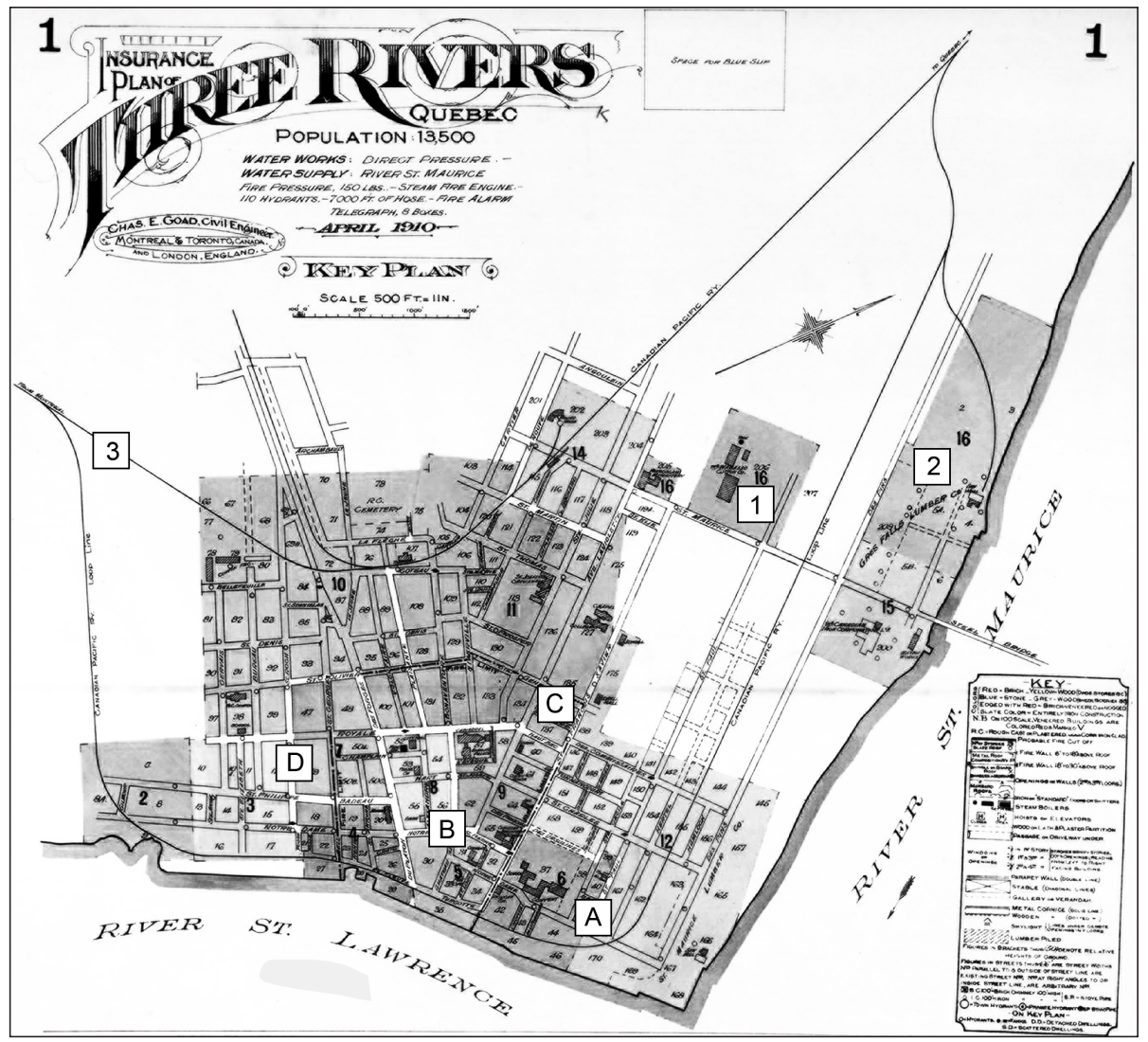

I.Wabasso Cotton Co. - 2. Grès Falls (premier emplacement) - 3. Chemin de fer du Canadien Pacifique

A. Quartier Ste-Ursule - B. Quartier St-Louis - C. Quartier Notre-Dame - D. Quartier St-Philippe Source: Bibliothèque et archives nationales du Québec (BanQ-Vieux-Montréal), Collection numérique «Cartes et plans» Insurance plan of Three Rivers, Quebec, Montreal; Toronto: Chas. E. Goad, 1910. 


\section{Figure 3}

\section{Plan de la ville de Trois-Rivières en 1930: Quartiers et principales industries}

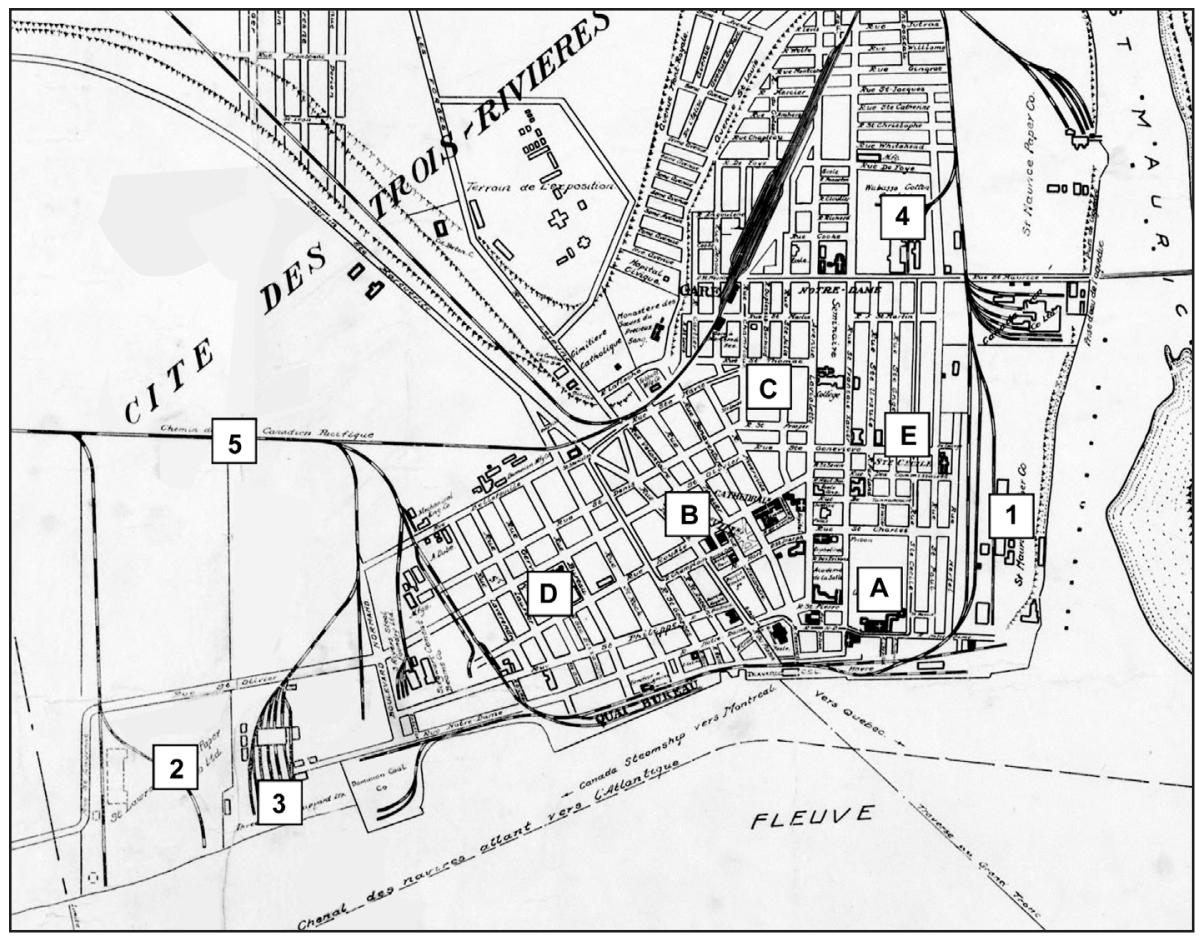

I. Canadian International Paper - 2. St. Lawrence Paper Mills - 3.Three Rivers Shipyard Co. Ltd. - 4. Wabasso Cotton Co. - 5. Chemin de fer du Canadien Pacifique

A. Quartier Ste-Ursule - B. Quartier St-Louis - C. Quartier Notre-Dame - D Quartier St-Philippe - E. Quartier Ste-Cécile

Source: Bibiliothèque et Archives Canada (BAC), Carte des cités des Trois-Rivières et du Cap-dela-Madeleine. Compilée et préparée par Roméo Morrissette, I.C., Ingénieur de la cité du Cap-dela-Madeleine (1930).

sifié $^{33}$. Ainsi l'architecture des maisons change radicalement. Non seulement des quartiers anciens doivent être rebâtis après l'incendie de 1908, mais dans les nouveaux quartiers ouvriers, les maisons basses à mansardes laissent place à des constructions cubiques, à étages et toits plats ${ }^{34}$.

33. R. Hardy et N. Séguin, Histoire de la Mauricie, op. cit., 591 et 595.

34. A. Gamelin, R. Hardy et al., Trois-Rivières illustrée, op. cit., 109-112; R. Hardy et N. Séguin, Histoire de la Mauricie, op. cit., 591-600. 


\section{Le Cap-de-la-Madeleine}

Avant 1870, «les perspectives d'expansion industrielle sont nulles au Capde-la-Madeleine ${ }^{35} »$. Ainsi, début du $\mathrm{XX}^{\mathrm{e}}$ siècle, le Cap-de-la-Madeleine conserve un visage essentiellement rural. Quelques logements ouvriers y sont toutefois présents à la suite de la première industrialisation et du développement du commerce du bois:

Depuis les ponts du Saint-Maurice, le village se continue presque sans interruption le long de la rivière, et ensuite le long du fleuve Saint-Laurent jusqu'au-delà de l'église du Cap de la Madeleine. La plupart des maisons appartiennent à des ouvriers; elles sont toutes de bois, à un seul étage et bien proprettes $^{36}$.

Lorsque les Oblats arrivent dans la localité en 1902, ils parlent des «chemins étroits et sablonneux du $\mathrm{Cap}^{37}$ ». La population atteint alors à peine 1500 personnes. L'implantation de la Grès Falls, en 1910, et l'ouverture de la Wayagamack Pulp and Paper Co. dans l'île de la Potherie un an plus tard marquent un changement important, modifiant la physionomie de la municipalité. En vingt ans, la population de l'agglomération triple, atteignant 6738 personnes en 1921, puis 8748 en $1931^{38}$. Les travailleurs affluent, les maisons de pensions qui logent les ouvriers sont saturées et les habitations manquent. C'est un véritable quartier ouvrier qui prend forme sur la rive du Saint-Maurice. En 1918, de nouvelles rues sont ouvertes, les anciennes sont prolongées et, un an plus tard, le maire et ses conseillers demandent au gouvernement du Québec une part de l'aide financière du gouvernement fédéral pour les logements ouvriers ${ }^{39}$.

Les ouvriers qui habitent au Cap travaillent pour la plupart à la St. Maurice Paper Co. et à la Wayagamack. Celle-ci «emploie, à ses usines seulement, plus de mille ouvriers, dont la plus grande partie résident au Cap-de-la-Madeleine ${ }^{40} »$. Au cours de ces années, le Cap va changer plusieurs fois de statut, gagnant sans cesse en importance. Ainsi, en 1915 a lieu la scission municipale entre Sainte-Marie-du-Cap-de-la-Madeleine, à

35. Maurice Loranger, Histoire de Cap-de-la-Madeleine (1651-1986) (Cap-de-la-Madeleine, s.n., 1987), 171.

36. Napoléon Caron, Deux voyages sur le Saint-Maurice (P.V. Ayotte, Trois-Rivières, 1889), 311-312, cité dans S. Rousseau, Trois-Rivières : une ville dépossédée..., op. cit., 128.

37. François De Lagrave, Cap-de-la-Madeleine, 1651-2001: une ville d'une singulière destinée (Cap-de-laMadeleine, Les Éditions du $350^{\mathrm{e}}$ anniversaire, 2002), 340.

38. R. Hardy et N. Séguin, Forêts et société en Mauricie..., op. cit., 196.

39. F. De Lagrave, Cap-de-la-Madeleine, 1651-2001..., op. cit., 501-505, 539-543.

40. BAnQ Trois-Rivières, Fonds Cour Supérieure (TP11), S3, SS2, 1918, n 172: Wildy St Cyr vs Wayagamack Pulp and Paper Co. 
l'ouest, et Sainte-Marthe-du-Cap, à l'est. Cette scission est essentiellement motivée par la hausse des impôts et des dépenses municipales pour l'aménagement de l'agglomération (trottoirs, électrification, aqueduc, aménagement d’une salle municipale): «Cap-de-la-Madeleine ne désirait plus être une simple campagne, mais aspirait à un autre statut municipal ${ }^{41}$.» Dès 1917, les élus entament des démarches afin d'ériger la municipalité en ville. En février 1918, les nouveaux statuts sont accordés à la ville du Cap-de-la-Madeleine. En 1920, le Cap demande cette fois d'accéder au statut de Cité, ce qui lui est accordé deux ans plus tard ${ }^{42}$.

Le plan de 1930 (figure 4) montre une agglomération du Cap-de-laMadeleine, certes modeste, mais qui n'est plus une petite bourgade rurale. La rue Notre-Dame, qui longe le Saint-Maurice puis le fleuve pour arriver au Sanctuaire, et la rue Fusey sont à présent reliées par un quadrillage de rues. Le long du fleuve, la St. Maurice Paper est installée directement à droite du Sanctuaire des Pères Oblats. La Tidewater Shipbuilders Ltd. a établi en 1917 son chantier naval le long du Saint-Maurice, à hauteur de l'île Caron. Le chemin de fer qui relie ces deux industries et la Wayagamack Pulp and Paper au réseau du Canadien Pacifique traverse en diagonale la municipalité. À proximité de la Tidewater se distingue l'église de la nouvelle paroisse de la Sainte-Famille, créée en raison de l'augmentation rapide de la population.

Cette brève étude de l'urbanisation de Trois-Rivières et du Cap-de-laMadeleine après l'explosion démographique ayant accompagné l'arrivée de la grande industrie à l'embouchure du Saint-Maurice montre donc deux agglomérations subissant d'importants changements en l'espace d'une dizaine d'années. Quant aux usines, elles doivent, lors de leur implantation, s'accommoder d'un réseau urbain préexistant. Elles vont également devoir faire face à d'autres usages du territoire, voire à d'autres conceptions du "progrès» et de la modernité.

\section{ATTIRER LES INDUSTRIES EN VILLE: LA CROISSANCE À TOUT PRIX? DES VOIX DISSONANTES}

$\mathrm{Si}$, dans la presse, les réactions à l'installation de nouvelles industries sont majoritairement enthousiastes, une thématique apparaît rapidement: les émanations incommodantes des usines de pâtes et papiers. "Pas de publicité à l'envers» titre Le Bien Public en avril 1914:

41. F. De Lagrave, Cap-de-la-Madeleine, 1651-2001..., op. cit., 515.

42. Ibid., 491-493, 515, 524, 561 ; M. Loranger, Histoire de Cap-de-la-Madeleine..., op. cit., 176-183 ; R. Hardy et N. Séguin, Histoire de la Mauricie, op. cit., 619-620. 
Figure 4

\section{Plan de la ville du Cap-de-la-Madeleine en I930: Principaux édifices et industries}

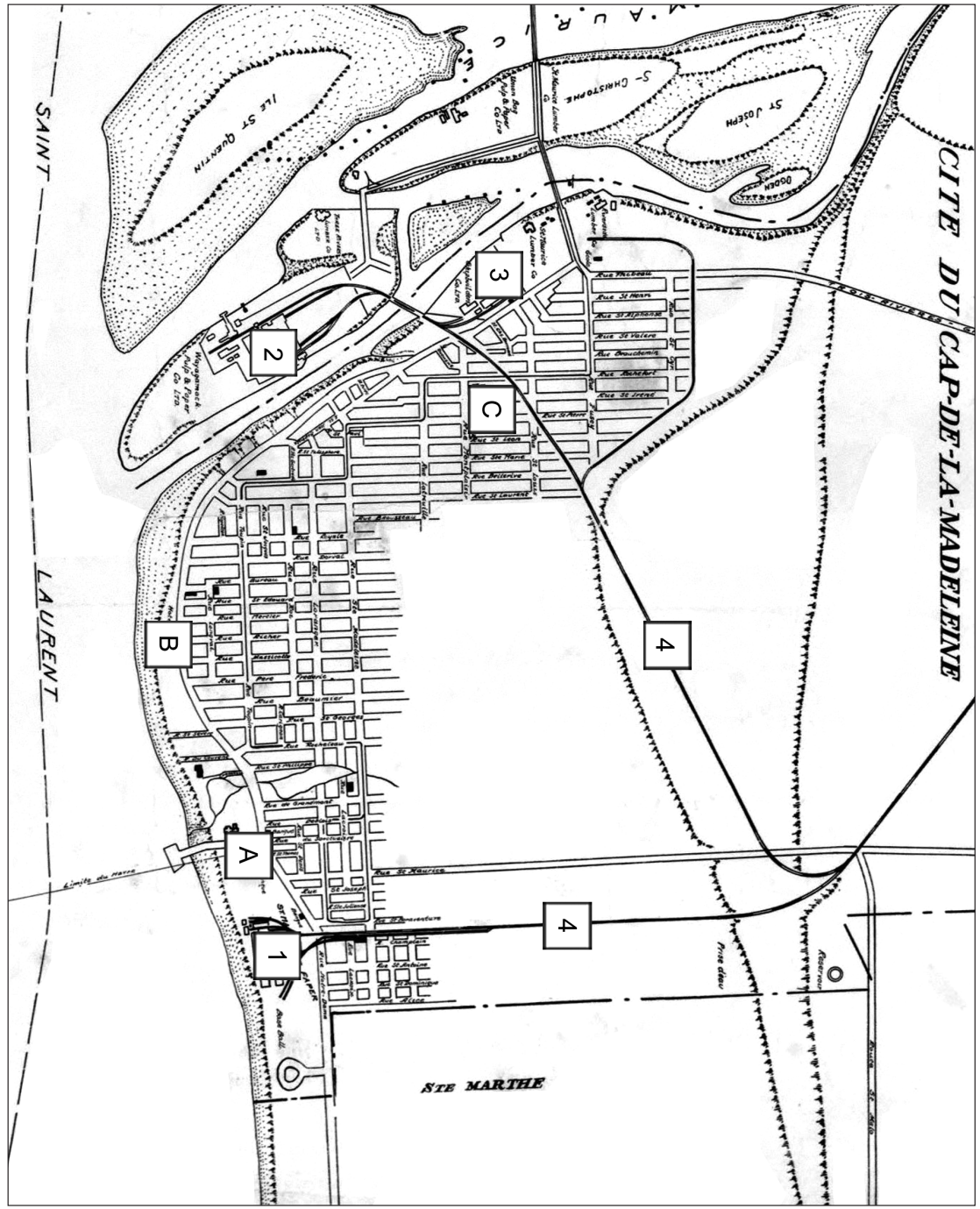

I. St. Maurice Paper Co. - 2. Wayagamack Pulp and Paper Co. - 3.Tidewater Shipbuilders Ltd. - 4. Chemin de fer du Canadien Pacifique

A. Sanctuaire Notre-Dame du Cap - B. Hôtel de ville - C. Église Ste Famille Source: BAC, Carte des cités des Trois-Rivières et du Cap-de-la-Madeleine. Compilée et préparée par Roméo Morrissette, I.C., Ingénieur de la cité du Cap- de-la-Madeleine, 1930. 
Nous avons été les premiers à réclamer pour la prospérité de notre cité, le précieux apport d'une publicité bien entendue, et faite à propos. L'odeur nauséabonde qu'émane trop copieusement vers nous la grande pulperie de l'Ile [Wayagamack], nous en fait une publicité: mais c'est de la publicité à l'envers, et de celle qu'il ne faut pas $^{43}$.

Ces industries qui s'installent dans un tissu urbain préexistant entrent à certains égards en concurrence, voire en conflit, avec d'autres usages, d'autres fonctions urbaines, par rapport à une ville créée par et pour l'industrie. Dans ce cas-ci, les nuisances agissent comme un révélateur des frictions entre ces différents usages.

C'est au Cap-de-la-Madeleine que les résistances sont les plus nombreuses. Des habitants intentent des procès à la Wayagamack Pulp \& Paper Co. en raison des émanations qui dégradent leurs propriétés, tandis que les Pères Oblats du Sanctuaire Notre-Dame font appel au Conseil d'hygiène concernant les émanations de la St. Maurice Paper Co.; les autorités appuient certaines de ces revendications tandis que les Annales $d u$ Très Saint Rosaire ${ }^{44}$ font régulièrement, dans leurs pages, la liste des doléances à l'encontre des industries papetières.

$\mathrm{Au}$ Cap, plus qu'à Trois-Rivières, les nuisances de ces industries gênent une activité essentielle pour la municipalité : le tourisme et la visite de nombreux pèlerins au Sanctuaire. Celui-ci est effectivement une attraction non négligeable dans la région, attirant plusieurs dizaines de milliers de visiteurs chaque année. Dans une lettre adressée au Conseil d'hygiène, le père Valiquette s'inquiète : «Cette peste éloigne les pèlerins, les touristes, menace nos arbres d'ornementation. Nous avons sacrifié plusieurs milliers de piastres pour rendre notre lieu de pèlerinage agréable et attrayant, mais avec cette peste, la vie ici devient intolérable ${ }^{45}$.»

Le Cap était, avant la venue des industries papetières, encore à caractère majoritairement rural, comme nous l'avons déjà évoqué. L’industrialisation a certes apporté des éléments très positifs, dont tous, y compris les Pères Oblats, se réjouissent. L'aménagement des routes, des trottoirs, de l'électricité, des lignes de chemin de fer profite en effet autant à l'industrie qu'au

43. "Pas de publicité à l'envers ", Le Bien Public, 30 avril 1914, 1.

44. Périodique fondé en 1892 par les pères Oblats du Cap. Il portera ensuite le nom d'Annales de Notre-Dame du Cap (1919-1967), pour être aujourd'hui nommé Revue Notre-Dame-du-Cap.

45. Lettre du Révérend Père Valiquette: BAnQ Québec, Fonds Conseil d’hygiène (E88), Rapports d'inspections et décisions du Conseil d'hygiène de la province de Québec, vol. XXV, 1917, Pollution de l'air atmosphérique par des gaz provenant d'une usine, p. 189-196. 
tourisme. Cependant, les odeurs, elles, sont à cet égard contre-productives, la «brise parfumée et vivifiante de jadis ${ }^{46}$ » faisant à présent défaut.

En plus d'impliquer un usage divergent de l'espace, la forte présence et influence religieuse au Cap insuffle des idées contrebalançant la course au progrès dans laquelle se lancent de nombreuses municipalités. Les Oblats du Cap-de-la-Madeleine se présentent souvent comme les garants de la moralité des abords du Sanctuaire. Dans les Annales du Très-Saint Rosaire, notamment, on retrouve plusieurs thématiques mêlant ultramontanisme, régionalisme trifluvien et promotion d'un progrès moral ${ }^{47}$. Une certaine polarisation entre clergé et industries subsiste sur cette base jusqu'au début du XX $\mathrm{XX}^{\mathrm{e}}$ siècle. À ces revendications se greffe la défense du français par rapport à la langue anglaise, et plus largement, des francophones face à l'invasion du Québec par les capitaux anglais et américains qui en mobiliseraient les principales ressources. Le développement régional est ainsi prôné afin d'éviter l'implantation du capital étranger, ou pire, le départ des forces de travail pour l'Ontario ou les États-Unis ${ }^{48}$.

Bref, dans ce sillage, une certaine presse est plutôt encline à prendre les industriels à partie, sur la thématique des émanations entre autres:

Tous ont aussi constaté que nos propriétaires de pulperies n'ont pas encore voulu prendre le moyen d'empêcher leurs émanations fétides d'empester les habitants de la région et ceux qui viennent la visiter. Nos bons journaux, le «Devoir», l'«Action Catholique», le «Droit», «Le Bien Public» font une campagne contre ces empoisonneurs. Nous leur en savons gré ${ }^{49}$.

Ce discours est donc soutenu par une partie de la presse trifluvienne. Ainsi, Le Bien Public, présentant ces mêmes caractéristiques (catholique, conservateur et pro-francophone $)^{50}$, évoque plusieurs fois les déconvenues touristiques liées aux odeurs des pulperies et soulève la mauvaise image que cela donne de la ville aux visiteurs extérieurs ${ }^{51}$, «emportant de TroisRivières le souvenir contrariant d'une ville qui ne sent pas bon» et don-

46. Annales du Très-Saint Rosaire, septembre 1917, 325.

47. F. De Lagrave, Cap-de-la-Madeleine, 1651-2001..., op. cit., 343-345, 551-555.

48. R. Verrette, Les idéologies du développement..., op. cit., 26-32, 213-216.

49. Annales de Notre-Dame du Cap, novembre 1922, 325.

50. R. Hardy et N. Séguin, Histoire de la Mauricie, op. cit., 712-714, 719-721, 804-808; Maude RouxPatte, Le Bien Public (1908-1978). Un journal, une maison d'édition, une imprimerie (Québec, Septentrion, 2013), $210 \mathrm{p}$.

51. "Ça sent le Jambon... », Le Bien Public, 30 avril 1914, 1 ; «Pas de publicité à l’envers », Le Bien Public, 30 avril 1914, 1; «La Wayagamack et ses odeurs », Le Bien Public, 6 juillet 1916, 1. 
nant à la ville une «réputation détestable ${ }^{52}$ ». Le journal commente l'arrivée de la St. Lawrence Paper, à l'ouest de la ville, comme suit:

En supposant que rien ne soit fait pour remédier à la situation dont on nous menace, notre vieille ville des Trois-Rivières vivra dans une continuelle puanteur. Quand la fumée et les odeurs d'œufs pourris ne seront pas soufflés [sic] sur nous de l'Est, ce sera de l'Ouest qu'on nous les soufflera. Et ce ne sera ni plus gai, ni meilleur ${ }^{53}$.

Le journal ne manque d'ailleurs aucune occasion de prendre à partie les autorités de la ville et les autorités sanitaires pour leur inaction contre les pulperies. En Une d'un autre journal, Le Courrier, la rubrique "Je dis tout » exprime à plusieurs reprises l'inquiétude de voir la ville décliner si la situation persiste: "Ces odeurs doivent disparaître, sans quoi, c'est la ruine de notre ville ${ }^{54}$.» Force est cependant de constater que les odeurs de la ville deviennent l'une de ses caractéristiques principales, comme le montre le récit de voyage de Maurice Genevois qui, en 1939, décrira TroisRivières ainsi:

Quarante mille habitants à peu près, une profusion d'escaliers extérieurs, les montagnes de billots sur les quais, et surtout l'odeur des pulperies: un relent de pourriture qui traînaille dans l'air des rues, un peu aigre, un peu fétide ${ }^{55} \ldots$

Mais si ces cheminées qui fument sont désagréables aux visiteurs, elles sont également vues comme le signe de la prospérité de la ville ${ }^{56}$. L'Almanach de 1912 en témoigne:

Voyez les hautes cheminées qui fument constamment, entendez les coups de sifflet stridents, voyez le va-et-vient dans les rues, les mille et un bruits de l'activité humaine qui se meut constamment dans toutes les branches de la vie. [...] C'est l'image de la ville industrielle, de la ville entreprenante ${ }^{57} \ldots$

Ces deux discours opposés posent une question: celle de la destination de la ville, de l'arbitrage à effectuer entre différentes fonctions que lui prêtent des acteurs distincts.

52. "La Wayagamack et ses odeurs», loc. cit., 1.

53. "La pollution de l'air», Le Bien Public, $1^{\text {er }}$ juin 1922, 1.

54. "Je dis tout", Le Courrier, 9 juin 1914 et 12 juin 1914, 1.

55. Cité dans F. De Lagrave, Cap-de-la-Madeleine, 1651-2001..., op. cit., 556.

56. Discours également relevé par des chercheurs européens: Stephen Mosley, The Chimney of the World. A History of Smoke Pollution in Victorian and Edwardian Manchester (Londres-New York, Routledge, 2008), 271 p. ; G. Massard-Guilbaud, Histoire de la pollution industrielle..., op. cit., 63-114.

57. Almanach de 1912, 64, cité dans R. Verrette, Les idéologies du développement..., op. cit., 196. 
Une autre fonction attendue de la ville, il va sans dire, est sa fonction résidentielle. Et là aussi, les frictions ne tardent pas à apparaître. Si l'ensemble des citoyens subissent les mauvaises odeurs, certains sont aux premières loges des émanations, lesquelles dégradent leurs propriétés et les empêchent d'en jouir pleinement. Plusieurs habitants du Cap-de-laMadeleine qui subissent directement les fumées provenant de la Wayagamack déposent des procédures devant la Cour supérieure de Trois-Rivières entre 1917 et 1925. Les terrains des plaignants sont situés majoritairement aux abords de la rivière Saint-Maurice, et donc en face de la Wayagamack. Cependant, la défense de l'industriel, parmi d'autres arguments, souligne que les résidents qui se plaignent habitent

un quartier essentiellement manufacturier et qui a son existence suite à la présence des usines de la défenderesse, et que les opérations des dites usines, loin d'être une nuisance pour le demandeur constitue [sic] un avantage pour lui. [...] S'il résulte des inconvénients par suite de l'exploitation des dites usines, ce sont ceux dont tout voisin doit souffrir, et particulièrement tout résidant [sic] d'un quartier manufacturier comme celui en question ${ }^{58}$.

Les dirigeants de la Wayagamack estimaient en effet que, ayant permis le développement de la ville du Cap, l'augmentation du nombre de ses habitants, et par conséquent la hausse des loyers et de la valeur du foncier, les nuisances subies par les citoyens étaient largement compensées, et qu'ils tiraient plus de bénéfices que d'inconvénients de la présence de cette industrie. Ces citoyens, nous le verrons, obtiendront finalement des dédommagements.

Mais cette problématique, le fait de subir des nuisances sans en tirer des bénéfices directs, n'en sera pas moins évoquée à plusieurs reprises, notamment, une nouvelle fois, dans Le Bien Public. Ainsi, lorsque le président du Bureau d'hygiène compare la situation trifluvienne à celle de la Scandinavie, le journal rétorque :

Une chose intéressante [...] serait celle de savoir si en Suède ou en Norvège on a laissé les puantes pulperies s'établir en plein centre habité, fonctionner en liberté au milieu d'une population dense, comme on a osé faire la chose ici aux Trois-Rivières. Nous croyons plutôt que c'est le contraire qui est arrivé et que les pulperies et leur peste ont été reléguées assez loin dans la forêt pour

58. BAnQ Trois-Rivières, Fonds Cour Supérieure (TP11), S3, SS2, 1922, $\mathrm{n}^{\circ} 440$ : J.H. Leduc vs Wayagamack Pulp and Paper Co. 
ne pas incommoder les gens qui ne vivent pas exclusivement de la fabrication du papier $\mathrm{kraft}^{59}$.

Une affaire alimente ces frictions : un autre procès qui se tient à Montréal entre A. J. Brown et la Canada Paper Co. ${ }^{60}$. Le plaignant est propriétaire d'une maison de villégiature à Windsor Mills et affirme que, depuis les changements de procédés de fabrication de la Canada Paper Co., située non loin de sa propriété, il ne peut plus jouir de celle-ci, les odeurs et la poussière l'ayant rendue inhabitable. À la différence des habitants du Capde-la-Madeleine, Brown ne se contente pas de demander des dédommagements, il réclame également une injonction contre la compagnie pour qu'elle soit obligée de changer de procédé de fabrication sous peine de fermeture $^{61}$. L'industriel tente une défense similaire à celle plaidée par la Wayagamack Plup and Paper devant la cour de Trois-Rivières :

The town of Windsor is essentially an industrial centre, having a population of about 2200 inhabitants, and is almost entirely dependent on the operation of the defendant's mills for its prosperity and the support of its citizen, nearly three-quarters of the labour of the Town working for the defendant ${ }^{62}$.

Les habitants seraient habitués aux odeurs et n'en seraient pas incommodés, seuls les visiteurs occasionnels et les résidents d'été les trouveraient dérangeantes ${ }^{63}$. Les dirigeants des industries papetières, notamment à Trois-Rivières, se mobilisent. Des aides financières sont décidées en assemblée, à la Wayagamack Pulp and Paper, pour aider la Canada Paper Co. à faire face aux frais de justice ${ }^{64}$. Une décision judiciaire en faveur de Brown présenterait en effet une menace, celle de voir la justice prohiber certains modes de fabrication jugés nocifs ou simplement incommodants. Cela établirait un précédent juridique inquiétant, pouvant entraîner des

59. "La peste des pulperies», Le Bien Public, 7 septembre 1922, 1.

60. "Try to Settle Sulphate Smell», Pulp and Paper Magazine, 22 avril 1920, 434; «Le tribunal accorde une injonction», La Tribune (Sherbrooke), 2 juillet 1920, 3 ; «Un jugement important pour Windsor Mills», La Tribune (Sherbrooke), 9 février 1922, 1; "Ces odeurs de la Wayagamack et de la St. Maurice Paper», Le Bien Public, 19 septembre 1918, 1; «Les fumées de la Wayagamack nuisent à la navigation », Le Bien Public, 16 octobre 1919, 1 ; J.-P. Charland, Les pâtes et papiers du Québec..., op. cit., 83; Guy Moreau, Histoire de Windsor et ses environs (Windsor, 1997), $427 \mathrm{p}$.

61. Sur les recours en justice, et les demandes de dommages ou d'injonction, voir Noga MoragLevine, Chasing the Wind. Regulating Air Pollution in the Common Law State (Princeton-Oxford, Princeton University Press, 2003), 87-102.

62. BAnQ Vieux-Montréal, Fonds Cour du banc de la reine (TP9), S2, SS5, SSS4, Jugements portés en appel à la Cour Suprême et au Conseil Privé, 1921-1925, n 143: Alain Joseph Brown vs Canada Paper Company.

63. "Try to Settle Sulphate Smell», Pulp and Paper Magazine, 22 avril 1920, 434.

64. BAnQ Vieux-Montréal, Fonds Consolidated Bathurst Incorporated (P149), Minutes of the meetings of the board of Directors of the Wayagamack Pulp and Paper Co. Ltd., 26, vol. 3, 1920, p. 33. 
fermetures d'usines. Finalement, Brown gagnera sa cause, obtenant un dédommagement de 15000 \$ et l'injonction demandée.

Cette décision, si elle effraie les industriels par sa jurisprudence, est accueillie favorablement par le Bien Public. Plusieurs articles soulignent le fait que la grande industrie ne se situe plus à présent au-dessus des lois et doit respecter le droit de tous de bénéficier d'un air pur. Ainsi, lorsque les autorités trifluviennes, pour justifier leur inaction, déclarent que la nocivité des émanations n’a pas été prouvée, Le Bien Public s’insurge : l'odeur a suffi à Windsor Mills. Mais d'ajouter:

Sans doute que tous les citoyens n'ont pas les moyens de défendre leur droit comme a pu le faire le millionnaire Brown. Celui-ci pouvait se payer le luxe d'avoir de l'air pur à sa maison de campagne. La justice de ce pays lui a reconnu ce droit ${ }^{65}$.

Tous ne pourraient donc faire valoir leurs droits de la même manière. De plus, toujours selon ce journal, c'est la faillite de la technique à trouver un remède aux émanations qui pousse les citoyens à se tourner vers les tribunaux:

Il est possible que la science n'ait pas réussi à empêcher la compote de bois préparée au sulfate de sentir mauvais, et qu'aucun moyen de supprimer l'odeur n'ait été trouvé jusqu'en 1921; ceci n'est qu'une faillite de la science. Mais il n'est pas exact de dire qu'il n'existe aucun moyen de supprimer les senteurs des pulperies. Tous nos tribunaux du pays l'ont récemment trouvé ce moyen ${ }^{66}$.

Là où les fumées peuvent être vues comme le signe du progrès, Le Bien Public y voit un échec: «Est-ce que ce serait là l'emblème du progrès moderne ${ }^{67}$ ?"; "Dans toutes les villes un peu modernes on s'applique à conserver à l'air toute sa pureté et toute sa salubrité ${ }^{68}$. "Si la science est tenue en échec et que l'accès à la justice n'est réservé qu'à une minorité, qui peut prendre la défense de l'ensemble des habitants? Au Cap-de-laMadeleine, le conseil municipal semble avoir été attentif à la problématique des gaz, mais se montre plus sévère envers la Wayagamack qu’envers la St. Maurice Paper. Cette attitude peut s'expliquer: la St. Maurice a été autorisée et soutenue financièrement par la municipalité et participe à son développement. Cependant, la Wayagamack, qui empeste aussi le Cap-

65. «Notre Société médicale et ces gaz infects», Le Bien Public, 23 novembre 1922, 1.

66. "La peste des pulperies», Le Bien Public, 7 septembre 1922, 1.

67. "Courrier de la cité: de l'hygiène», Le Bien Public, 28 juin 1917, 8.

68. "Pour supprimer l’odeur et la fumée», Le Bien Public, 14 septembre 1922, 1. 
de-la-Madeleine, est située théoriquement en territoire trifluvien. La difficulté d'intervention dans ce contexte n'échappe pas aux autorités. Dans le procès-verbal du 23 novembre 1920 qui motive la demande du statut de Cité pour le Cap, il est dit explicitement que «La ville devrait avoir le privilège de statuer, règlementer et défendre l'émanation des gaz, fumées, etc., qui s'échappent des usines en dehors de la ville et qui envoient leur gaz sur la ville ${ }^{69}$. Ces tensions entre habitants et industriels autour de la question des gaz et fumées font émerger plusieurs questions dans l'espace public: Qui a le droit de se plaindre légitimement des gaz? Ceux qui ne jouissent pas directement de l'industrie? Ceux qui sont dans une habitation dont le seul usage est la villégiature? Tout le monde a-t-il les moyens de faire respecter également ses droits?

En plus d'entraver les visées touristiques et la fonction résidentielle des municipalités, les émanations nuisent également à une autre activité économique de la ville: la vie portuaire. L'implantation de la St. Lawrence Paper à l'ouest de la ville, bloquant le développement du port, provoque des inquiétudes en 1920. Mais les gaz et fumées ont également un impact sur la navigation. Dès 1919, le département fédéral de la Marine écrit à la Chambre de commerce des Trois-Rivières et à la Commission du Havre à la suite d'une plainte des Pilotes du Saint-Laurent. La navigation à proximité de l'île de la Potherie serait parfois gênée par la fumée dégagée par les cheminées des usines de pâtes et papiers de Trois-Rivières et en particulier de la Wayagamack et de la St. Maurice Paper. Les premières plaintes remonteraient à juillet $1917^{70}$ et ces troubles se présenteraient tous les étés $^{71}$. L'État fédéral étant compétent en matière de marine et de pêcheries, il peut intervenir auprès des industriels lorsque ceux-ci mettent en péril les pêcheries (rejets causant des pertes en populations de poissons ${ }^{72}$ ) ou la navigation, dans le cas ici étudié ${ }^{73}$.

En 1922, une commission étudie la plainte portée devant le gouvernement fédéral par les Pilotes du Saint-Laurent ${ }^{74}$. Une réunion est tenue à

69. F. De Lagrave, Cap-de-la-Madeleine, 1651-2001..., op. cit., 561.

70. «Les fumées de la Wayagamack nuisent à la navigation », Le Bien Public, 16 octobre 1919, 1.

71. "Les odeurs sur la ville», Le Nouvelliste, 22 février 1922, 1.

72. Au moins deux actions ont d'ailleurs été menées par l'inspecteur des pêcheries de Montréal pour mise en danger des populations de poissons par des rejets de substances délétères par la Wayagamack en 1912 et 1913 (BAnQ Trois-Rivières, Fonds Cour du banc de la reine (TP9), S3, SS29, SSS1, 1913, Dossier 304 : Joseph Riendeau vs The Wayagamack Pulp and Paper Co.).

73. Jacques Gosselin, "Le Droit à l'environnement en droit québécois et canadien», dans Pierre Gosselin, dir., Santé environnementale au Québec, Bases théoriques et pratiques (Québec, Les Publications du Québec, 1986), 195-242.

74. «Une enquête sur la fumée des pulperies», Le Nouvelliste, 3 mars 1922, 1. 
Trois-Rivières en présence des dirigeants de la Wayagamack et de la St. Maurice Paper Co. et une entente est finalement conclue : les chimistes du département de la Marine et ceux des industries papetières se rencontreront afin de venir à bout de ces fumées dangereuses ${ }^{75}$. Alors qu'en septembre Le Bien Public laisse entendre que les plaintes de la corporation des Pilotes ne donnent aucun résultat ${ }^{76}$, des rencontres ont lieu entre les autorités de la St. Maurice et des représentants du département de la Marine ${ }^{77}$.

En 1924, la Wayagamack Pulp and Paper et la St. Maurice Paper entreprennent toutes deux des travaux et des changements dans leurs modes de production afin de réduire les émanations de fumées. Et les dirigeants de la Wayagamack ne citent qu'une seule raison à ces aménagements: "Any difficulty we might have with the Government owing to the question of navigation would be completely done away with when the whole unit was completed ${ }^{78}$. " Ce sont donc les autorités d'Ottawa qui font, ultimement, "bouger» les industriels, qui adaptent alors leurs modes de production. Certes, l'odeur ne disparaît pas totalement. Encore de nos jours, le fumet des usines à papiers parfume l'air de Trois-Rivières lorsque la météo y est propice. Cependant, les protestations, dans la presse comme auprès des tribunaux, baissent considérablement à compter du milieu des années 1920, laissant penser que ces aménagements furent efficaces.

La «cheminée qui fume ${ }^{79}$ » peut donc recouvrir différentes symboliques, selon la relation qu'a le citoyen à l'entreprise, selon son usage de l'espace urbain, selon sa situation géographique, plus ou moins proche de l'usine... $S$ 'il revient à la science de trouver des solutions techniques, la gestion de ces nuisances appartient aux autorités et aux tribunaux, chargés de trancher et d'effectuer l'arbitrage entre nuisances et croissance économique, dans l'intérêt d'une personne lésée ou du plus grand nombre. Sur cette question de l'intérêt général, un élément absolument décisif pour les autorités est de savoir si oui ou non ces émanations sont dangereuses pour la santé. L'inspecteur McCrady énonce dans un rapport envoyé au Conseil d'hygiène: "The main question immediately arises, "Is the degree of odor a

75. "Pulp Mills blamed for fogs», Pulp and Paper Magazine, 16 mars 1922, 205.

76. «Pour supprimer l’odeur et la fumée», Le Bien Public, 14 septembre 1922, 1.

77. BAnQ Vieux-Montréal, Fonds Consolidated Bathurst Incorporated (P149), Dossier «Smoke eliminating System».

78. BAnQ Vieux-Montréal, Fonds Consolidated Bathurst Incorporated (P149), Minutes of the Meetings of the board of directors of the Wayagamack Pulp and Paper Co. Ltd., 26, vol. 3, The $20^{\text {th }}$ day of March 1924.

79. G. Massard-Guilbaud, Histoire de la pollution industrielle..., op. cit., 8-9 ; St. Mosley, The Chimney of the World..., op. cit., 71-78. 
measure of the poisonous effect, as popularly regarded?" ${ }^{80}$ ». Que la "cheminée qui fume» soit synonyme de progrès ou d'incommodité, la question clé pour les autorités est de savoir si elle peut être synonyme de danger.

\section{D'INCOMMODANT À NUISIBLE: QUALIFIER LE DANGER}

Les impacts environnementaux et sanitaires de l'industrialisation et de l'urbanisation, fin $\mathrm{XIX}^{\mathrm{e}}$-début $\mathrm{XX}^{\mathrm{e}}$ siècle, ont surtout été approchés pour les dommages causés aux eaux, en lien avec l'une des préoccupations de l'époque: de l'eau salubre pour le plus grand nombre ${ }^{81}$. Et force est de constater que, dans cette lutte des autorités pour une eau saine, les rejets industriels ne figurent pas immédiatement en tête des préoccupations. Qu'en est-il de la pollution atmosphérique? La thématique est abordée dans plusieurs ouvrages sur les États-Unis. Ils traitent surtout de la pollution en ville, avec une attention particulière portée aux pollutions domestiques, aux fumées de charbon ou aux maladies professionnelles ${ }^{82}$. Une étude sur la pollution atmosphérique d'origine industrielle dans une ville mono-industrielle a cependant été réalisée à Sudbury, par Daniel Bouchard $^{83}$, tandis que R. A. Lucas, dans son ouvrage sur la vie dans les communautés mono-industrielles, consacre un paragraphe aux émanations subies par ces population ${ }^{84}$. Quant aux ouvrages qui s'intéressent à l'histoire des pâtes et papiers, ils accordent pour certains quelques pages

80. BAnQ Québec, Fonds Conseil d'hygiène de la province de Québec (E88), Rapports d'inspections et décisions du Conseil d'hygiène de la province de Québec, vol. XX, 1912, Vapours emanating from Wayagamack Pulp and Paper Co., p. 369-373.

81. F. Guérard, La santé publique..., op. cit., 90-179; Stéphane Castonguay et Dany Fougères, «Les rapports riverains de la ville: Sherbrooke et ses usages des rivières Magog et Saint-François XIX ${ }^{\mathrm{e}}$-XX ${ }^{\mathrm{e}}$ siècles", Revue d'histoire urbaine, XXXVI, 1 (automne 2007): 3-15; Stéphane Castonguay, «Les territoires de la pollution. L'environnement comme catégorie de l'action publique au Québec», dans Guy Massicotte, dir., Les Sciences du territoire (Québec, Presses de l’Université du Québec, 2008), 77-102; Michèle Dagenais, Montréal et l'eau, une histoire environnementale (Montréal, Boréal, 2011), 97-128; Gillis Peter, «Rivers of Sawdust: The Battle over industrial pollution in Canada, 1865-1903 ", Journal of Canadian Studies, 21, 1 (mai 1986): 84-103; Théodore Steinberg, Nature Incorporated. Industrialization and the Waters of New England (Cambridge, Cambridge University Press, 2003), 284 p.

82. À titre d'exemples: Walter E. Pittman Jr., "The Smoke Abatement Campaign in Salt Lake City, 1890-1925", Locus, 2, 1 (automne 1989): 69-78; Gerald Markowitz et David Rosner, Deceit and Denial. The Deadly Politics of Industrial Pollution (Berkeley, University of California Press, 2002), 408 p.; Angela Gugliotta, "How, When, and for Whom was smoke a problem in Pittsburgh?», dans Joël Tarr, dir., Devastation and Renewal. An Environmental History of Pittsburgh and its Region (Pittsburgh, Pittsburgh University Press, 2004), 110-125 ; David Stradling, Smokestacks and Progressives. Environmentalists, Engineers, and Air Quality in America, 1881-1951 (Baltimore-Londres, The Johns Hopkins University Press, 2002), 270 p. ; Frank Uekoetter, The Age of Smoke. Environmental Policy in Germany and the United States, 1880-1970 (Pittsburgh, University of Pittsburgh Press, 2009), 350 p.

83. Daniel Bouchard, Pollution, science et pouvoir. L'histoire d'un désastre écologique à Sudbury (1883-1945). Derrière l'écran de fumée, thèse de doctorat (histoire), Université d'Ottawa, 2003.

84. R. A. Lucas et L. Tepperman, Minetown, Milltown, Railtown..., op. cit., 95-102. 
aux pollutions provoquées par les usines, mais c'est souvent un bref récit factuel qui est proposé, agrémenté de quelques anecdotes ${ }^{85}$. Les débats soulevés par les fumées des usines papetières en termes de dangers sanitaires sont rarement évoqués.

Dans la controverse trifluvienne entourant ces émanations, peu de discussions concernent leur caractère incommodant. Tous y consentent: les fumées des usines de pâtes et papiers sentent mauvais, dégagent des poussières qui tachent le linge et recouvrent tout à l'intérieur des maisons si les fenêtres sont laissées ouvertes. Les discussions commencent lorsqu'il s'agit de définir le caractère corrosif de ces fumées. Les témoignages sont nombreux sur la dégradation du matériel : les Oblats se plaignent de l'atteinte aux objets de culte, les riverains de la détérioration de la peinture sur leurs maisons. Entre 1917 et 1925, 25 habitants du Cap-de-la-Madeleine mènent un total de 31 actions pour «dommages» contre la Wayagamack Pulp and Paper (certains initiant plusieurs procédures au fil des ans) ${ }^{86} . \mathrm{Si}$ tous les dossiers n'ont pas été conservés, les plumitifs et registres de sentence apportent quelques renseignements sur ces causes. Ainsi, les sommes réclamées vont de 150 \$ à 900 \$. Les plaignants demandent réparation pour de multiples dommages subis par leurs propriétés: peintures extérieures et intérieures souillées et corrodées, baisse des loyers ou de la valeur de la propriété, odeurs "nauséabondes», linge sali, dorures, cadres et meubles attaqués, tapis et bains souillés... Les sentences sont rendues "considérant que l'on ne saurait assigner d'autres causes à ces détériorations que les fumées et émanations gazeuses ${ }^{87}$ » et afin de couvrir le montant des travaux de peinture ainsi occasionnés. Cependant, la plupart des causes sont réglées à l'amiable, hors de la cour, empêchant de connaître les montants finalement perçus par les plaignants.

Les dommages matériels attribuables aux émanations sont donc peu à peu reconnus. Une étape supplémentaire dans la qualification de ces émanations comme nocives consiste à établir leurs impacts sur la végétation et sur la santé. La tâche s'annonce plus difficile. Un seul procès porté devant la Cour supérieure de Trois-Rivières demande dédommagement

85. A. Dion, L'industrie des pâtes et papiers..., op. cit., 178-182; J.-P. Charland, Les pâtes et papiers..., op. cit., 83-88.

86. BAnQ Trois-Rivières, Fonds Cour Supérieure (TP11), S3, SS2, 1917, $\mathrm{n}^{\circ} 226 ; 1918, \mathrm{n}^{\circ} 608,618$, 676,$172 ; 1919, \mathrm{n}^{\circ} 348,349 ; 1920, \mathrm{n}^{\circ} 610,611,47 ; 1921, \mathrm{n}^{\circ} 101,201,202,731 ; 1922, \mathrm{n}^{\circ} 440,517,518,649$, 650,$735 ; 1923, \mathrm{n}^{\circ} 595,618,315 ; 1924, \mathrm{n}^{\circ} 238,523,647 ; 1925, \mathrm{n}^{\circ} 388,389,468,469$. Liste dressée à l'aide des plumitifs de la Cour Supérieure de Trois-Rivières (BAnQ Trois-Rivières, Fonds Cour Supérieure (TP11), S3, SS2, SSS7). Les affaires dont les dossiers ont été conservés sont en italique.

87. BAnQ Trois-Rivières, Fonds Cour Supérieure (TP11), S3, SS2, 1918, $\mathrm{n}^{\circ} 172$ : Jugement rendu dans l'affaire Wildy St Cyr vs Wayagamack Pulp and Paper Co., le 11 novembre 1919. 
pour des dégradations subies par la végétation. Arthur Paquette, lui aussi habitant au Cap-de-la-Madeleine, réclame un dédommagement de $300 \$$ pour la mort de 6 arbres sur sa propriété, mort qu'il attribue aux gaz et fumées corrosives de l'usine ${ }^{88}$. La défense de l'industriel soulève les mêmes arguments que dans les autres causes, et y ajoute au sujet des arbres que ceux-ci "appartiennent à une famille ou espèce que le temps et l'âge affectent considérablement et [...] la défenderesse n'est nullement responsable de la déteriaration [sic] des dits arbres». De plus, "les grands froids de l'hiver précédent ont fait périr une grande quantité d'arbres dans toute la Province». La défense fait témoigner deux spécialistes: Henri Kieffer, ingénieur attaché au Service Forestier à Montréal, et Gustave Clodomir Piché, ingénieur forestier à Québec. La cause de Paquette est la seule cause relative aux émanations et portée devant le tribunal qui se solde par un rejet, le demandeur n'ayant "pas prouvé que les dommages subis par ses arbres ont été occasionnés par le fait non plus que par la faute de la défenderesse».

Cependant, si cet impact sur la végétation n'est pas officiellement reconnu, il est identifié par beaucoup comme un fait certain, et de là naît la crainte de l'impact des émanations sur la santé humaine. Un article du Bien Public énonce que si ces émanations ne nuisent pas à la santé,

la nature fait là une exception à ses lois. La nature nous met en garde contre les choses mauvaises, et la peste de certaines choses en décomposition suffit d'ordinaire à nous prévenir qu'il ne se trouve là rien de fameux pour la santé des gens. $[\ldots]$ nous admettons difficilement que les émanations de sulphate [sic] qui détériorent la peinture, corrodent le fer et anémient la végétation, ne produisent par contre aucun effet nocif contre l'humanité, cette plante si fragile et si délicate ${ }^{89}$.

Dans ce discours transparaît non seulement la théorie des miasmes ${ }^{90}$, encore très en vogue fin $\mathrm{XIX}^{\mathrm{e}}$ et début $\mathrm{XX}^{\mathrm{e}}$ siècle, établissant un lien direct entre mauvaises odeurs, putréfaction et nocivité pour la santé, mais l'être humain est aussi replacé au même niveau biologique que la faune et la flore qui l'entourent. Si ces dernières sont touchées, l'être humain ne saurait être épargné.

88. BAnQ Trois-Rivières, Fonds Cour Supérieure (TP11), S3, SS2, 1918, nº 676: Arthur Paquette vs Wayagamack Pulp and Paper.

89. "La peste des pulperies», Le Bien Public, 7 septembre 1922, 1.

90. Alain Corbin, Le miasme et la jonquille. L'odorat et l'imaginaire social aux XVIII et XIX ${ }^{e}$ siècles (Paris, Flammarion, 1986), 342 p. ; P. Thorsheim, Inventing Pollution..., op. cit., 10-18; G. Massard-Guilbaud, Histoire de la pollution industrielle..., op. cit., 69-72. 
Cette question de l'impact sur la santé fait aussi débat auprès des autorités sanitaires et médicales, et notamment au sein du Conseil d'hygiène. Une première enquête, menée par un chimiste, l'inspecteur McCrady, en 1912 , concluait que «it is my opinion that, although the odor may be strong in Three-Rivers, nevertheless the air cannot be considered poisonous ${ }^{91}{ }^{\prime}$. C'est sur cette base que vont s'appuyer tant le Conseil municipal de Trois-Rivières que le Bureau d'hygiène local ou les industries ellesmêmes pour calmer les craintes concernant la santé publique. Cependant, en 1917, le docteur Savary, lui aussi membre du Conseil d'hygiène, déclare dans son rapport sur les fumées de la St. Maurice Paper: "Ces gaz sont donc nuisibles et ne doivent pas être rejetés dans l'atmosphère ${ }^{92}$."

La comparaison de ces deux rapports témoigne de deux modes de preuves totalement différents entre le chimiste et le médecin. Le rapport de McCrady détaille la localisation de la Wayagamack Pulp and Paper Co., ses procédés de production, puis étudie la quantité de "vapeurs», leurs caractéristiques et leurs effets. Dans ce dernier paragraphe, le chimiste analyse les intervalles d'émission de ces fumées, les variations dans la direction des vents, la distance par rapport aux quartiers les plus peuplés de la ville, le taux de «dilution» des émanations dans l'air et la quantité de gaz alors présente par rapport à la quantité que l'on estime par ailleurs nocive. Dans ce cas, les données sont quantitatives, tandis que le docteur Savary opère de manière bien différente. Il décrit également, mais brièvement, les procédés et composés chimiques utilisés par la St. Maurice Paper, puis étudie les effets de ces gaz sur l'organisme. Mais plutôt que d'énoncer des données scientifiques et techniques, le médecin s'appuie sur des données beaucoup plus empiriques:

Les effets de ces vapeurs et de ces gaz ne se font pas également sentir chez tous les individus. Il va sans dire qu'ils sont plus sensibles chez les affaiblis. Chez ces derniers, ils produisent une sensation de faiblesse très prononcée, avec perte d'appétit, il survient des nausées allant parfois jusqu'au vomissement. On a aussi constaté assez souvent de la céphalalgie et de la rougeur des conjonctives.

91. BAnQ Québec, Fonds Conseil d'hygiène de la province de Québec (E88), Rapports d'inspections et décisions du Conseil d'hygiène de la province de Québec, vol. XX, 1912, Vapours emanating from Wayagamack Pulp and Paper Co., p. 369-373.

92. Rapport du Dr. Savary: BAnQ Québec, Fonds Conseil d'hygiène (E88), Rapports d'inspections et décisions du Conseil d'hygiène de la province de Québec, vol. XXV, 1917, Pollution de l'air atmosphérique par des gaz provenant d'une usine, p. 189-196. 
Il cite ensuite des procédés techniques qui permettraient de réduire ces émanations. Cette certitude de la nocivité basée sur l'expérience est également visible auprès des Pères Oblats: "plusieurs personnes se plaignent que cette peste fait grand tort à leur santé. Les uns ne peuvent dormir, les autres ont mal au cœur. Les yeux souffrent aussi de cette peste ${ }^{93}$.»

La question de la preuve de la nocivité des émanations pour la santé est donc un sujet sensible. Si la population qui l'éprouve quotidiennement semble établir un lien évident, les autorités sanitaires ont plus de difficulté à se prononcer. Cependant, tant les conseils municipaux que les bureaux d'hygiène sont composés des notables de la cité, dont certains eux-mêmes industriels. Leur mandat les appelle, en principe, à sauvegarder l'intérêt général. Mais quel est l'intérêt général? Lorsque l'Association médicale du district des Trois-Rivières discute des gaz et odeurs de la Wayagamack, les membres admettent "qu'il serait malséant de chercher à discréditer et à ruiner la plus grosse industrie de la ville ${ }^{94} »$. Mais pour Le Bien public, certaines priorités doivent subsister:

Il n'y a pas de doute que c'est bien de notre devoir, comme celui de tout le monde, de nous réjouir de la venue aux Trois-Rivières d'une nouvelle industrie très importante qui contribuera au progrès et au développement de la cité. Seulement, nous considérons que, même en face de cet avantage, nous ne devons jamais perdre de vue certaines garanties de sécurité qui sont au premier chef de l'intérêt public ${ }^{95}$.

Ce n'est finalement pas le débat sur la nocivité des émanations qui sera décisif dans la résolution de cette affaire, mais, comme nous l'avons évoqué, les entraves à la navigation, qui n'avaient aucun lien avec l'impact potentiel des fumées sur la santé. Ces controverses permettent cependant d'appréhender les différents discours et cadres mentaux relatifs aux émanations ainsi que les modes de preuves utilisés pour en démontrer le caractère éventuellement nocif.

93. Lettre du Révérend Père Valiquette: BAnQ Québec, Fonds Conseil d’hygiène (E88), Rapports d'inspections et décisions du Conseil d'hygiène de la province de Québec, vol. XXV, 1917, Pollution de l'air atmosphérique par des gaz provenant d'une usine, p. 189-196.

94. Archives du Séminaire Saint-Joseph (Trois-Rivières), Fonds Association médicale du district de Trois-Rivières, Rapports des réunions de l'Association médicale du district de Trois-Rivières du 6 mars 1905 au 24 avril 1913, Séance du 20 février 1913, p. 52-53.

95. “La pollution de l'air», Le Bien public, $1^{\text {er }}$ juin 1922, 1. 


\section{CONCLUSION}

$\mathrm{Au}$ début du $\mathrm{XX}^{\mathrm{e}}$ siècle, Trois-Rivières connaît un développement sans précédent après l'implantation, sur son territoire, de quatre usines à papier. De nouveaux quartiers émergent du côté trifluvien, tandis que le Cap-de-la-Madeleine, jusqu'alors essentiellement rural et peu développé, voit l'arrivée de plusieurs centaines d'ouvriers employés des usines papetières toutes proches. La cité trifluvienne préexistait à l'industrie, ce qui assure aux débats, lors de l'arrivée d'industries ou lors des nuisances à gérer, des forces en présence variées et équilibrées, qui s’expriment, débattent, se contredisent... Ces usines provoquent en effet des nuisances qui entrent en conflit, d'une part, avec la fonction résidentielle de la ville, et d'autre part avec ses activités touristiques et portuaires.

Ces frictions fournissent la possibilité d'étudier les discours liés à la ville, et notamment les questions d'arbitrage et de définition de l'intérêt général (croissance économique vs salubrité), mais également de percevoir des discours sur le territoire, l'espace urbain, ses limites, et le(s) «territoire(s) acceptable(s)» de la pollution industrielle au début du XXe siècle. S'il est question de la protection du milieu de vie, la question de la protection de l'environnement ne se pose que dans un but utilitaire. Implanter une usine en pleine forêt ne semble poser aucun problème, du moment que l'Homme n'en subit pas les nuisances. Les habitants se plaignent lorsque leurs propriétés sont touchées, leurs jardins d'agrément et d'ornementation dégradés. Les impacts sur la végétation ne sont évoqués que pour appuyer les craintes pesant sur la dangerosité de ces émanations pour l'organisme humain.

Certains discours ainsi étudiés font écho à des thèmes déjà rencontrés dans l'histoire des pollutions industrielles en Europe ou aux États-Unis: la double dialectique associant d'une part la fumée à un signe de progrès et de prospérité, et d'autre part, à une faillite de la science; ou encore le caractère inesthétique des émanations, qui acquièrent cependant au fil du temps une vocation quasi identitaire. De même, l'hésitation à qualifier ces fumées de dangereuses pour la santé est comparable. Si d'une part l'ancienne théorie des miasmes survit dans les esprits, des craintes s'expriment pour la santé humaine par assimilation et comparaison avec les dommages causés à la flore, d'autre part. Mais les thématiques étudiées, bien que rencontrant des discours observés à Manchester ou à Pittsburgh, par exemple ${ }^{96}$, s'inscri- $^{\prime}$ vent dans un cadre spécifiquement québécois aussi bien d'un point de vue

96. J. Tarr., Devastation and Renewal..., op. cit., 110-125 ; St. Mosley, The Chimney of the World..., op. cit., 69-117. 
légal et juridique, qu'en termes de gestion et d'acteurs impliqués. Transparaissent également, dans ces oppositions, des tensions inhérentes à la société québécoise, par exemple entre le clergé et l'industrie ou entre les industriels anglophones et la majorité francophone.

Cette étude met donc en évidence quelques lignes directrices, en termes de stratégies de gestion et d'argumentaires des différents acteurs notamment, qui mériteraient d'être nourries de nouvelles recherches avant de pouvoir conclure plus avant sur les «débordements industriels» et leur gestion au Québec. 